TRABAJO EN PROGRESO

\title{
Uso de interacciones humano-computadora para la recopilación de datos del proceso de lectura de comprensión de textos técnicos en inglés
}

\author{
Mayra Yadira Mejia-Sierra, Juan Sebastian Pinto- \\ Orduz, Maria Susana Avila-Garcia, Marco \\ Bianchetti, María Isabel Vázquez-de-la-Rosa
}

Publicado: 21 Septiembre 2016

\begin{abstract}
Resumen
Actualmente existen diferentes tipos de soluciones basadas en computadoras y dispositivos móviles que proveen opciones que asisten al usuario en el proceso de lectura, estas incluyen pero no se limitan a, definición de palabras desconocidas, subrayado de texto, escritura de notas, etc. Dado que el proceso de lectura de comprensión de un texto es un proceso cognitivo, la recolección de datos que nos permitan entenderlo resulta complejo. En este proyecto, el objetivo es recolectar datos sobre el proceso cognitivo de lectura de comprensión. Estas interacciones no pueden ser recolectadas ni analizadas por las aplicaciones de lectura disponibles actualmente. La información sobre el número de veces que una persona consulta la definición de una palabra, o las partes del texto resaltadas, el motivo para resaltar una frase, etc. siendo información de utilidad en la creación de nuevas estrategias para ayudar a la comprensión de textos técnicos en inglés.
\end{abstract}

Palabras clave: Sistema de Información; Comprensión de Textos Técnicos; Interfaz Humano-Computadora; Diseño Interactivo.

\section{Introducción}

En el ambiente académico, los profesores están interesados en que los alumnos desarrollen habilidades de lectura de comprensión de textos técnicos en inglés (TTI) permitiendo tener acceso a una vasta cantidad de material especializado. En [2] se reportaron las necesidades de información de algunos de los profesores del Departamento de Estudios Multidisciplinarios de la Sede de

\footnotetext{
Mejia-Sierra, MY., Avila-Garcia, MS., Bianchetti, M., Vázquez-de-laRosa, MS.

Departamento de Estudios Multidisciplinarios, Universidad de Guanajuato

Av. Universidad s/n Colonia Yacatitas, Guanajuato, México 38964 Email: my.mejiasierra@ugto.mx, susana.avila@ugto.mx,mb@ugto.mx de.rosasgonzalez@ugto.mx
}

Pinto-Orduz, JS.

Universidad Santo Tomás

Cra. 18 \#9-27, Bucaramanga, Santander, Colombia

Email: 85.jspo@gmail.com
Yuriria de la Universidad de Guanajuato, entre las que podemos encontrar preguntas de información como:

- SC_P2 ¿Qué porcentaje de las palabras técnicas nuevas fueron retenidas por el estudiante?

- SC_P3 ¿Qué porcentaje de palabras técnicas fueron nuevas para el estudiante?

- SC_P4 ¿Qué porcentaje de palabras no técnicas fueron nuevas para el estudiante?

- SC_P5 De las palabras que el estudiante ha visto anteriormente, ¿cuáles son las que no recordó el significado?

- SC_P6 ¿El estudiante reconoce una palabra pero no recuerda su significado en el contexto y cuántas veces la ha visto anteriormente?

- SC_P7 ¿Cuántas veces tuvo el estudiante que leer una palabra para retener el concepto dentro del contexto?

- I_P0 ¿Cuál es el nivel de inglés del estudiante?

- I_P7 ¿Cuál es el vocabulario técnico con el que cuenta el estudiante?

Estas necesidades de información son las que motivaron a la realización de este trabajo.

Siendo el proceso de lectura de comprensión de textos, un proceso cognitivo, la dificultad de recolectar datos del mismo es un reto. Tradicionalmente la lectura de textos se había realizado con materiales impresos, sin embargo, se ha demostrado que implementar las tecnologías de información en la lectura tiene efectos benéficos en el proceso de enseñanza-aprendizaje [1, 4] . Empleando interacciones humano-computadora (IHC) para la recolección de datos que permitan contestar a las necesidades de información del proceso de comprensión de textos expresadas por los profesores en [2] y así apoyar la creación de nuevas estrategias en la enseñanza de materias de carrera usando TTI.

Basados en estas necesidades, en este trabajo se analiza la lectura desde un punto de vista micro-estructural, es decir, en la lectura de comprensión a nivel vocabulario y se reporta la evolución de una prueba de concepto (PoC) que permita capturar datos del proceso de lectura y comprensión a través de IHC.

\section{Métodos}

Primero se realizó un análisis de los requerimientos y la funcionalidad inicial necesaria para una PoC. Para ello se consideró el análisis de la funcionalidad de 15 lectores 
electrónicos (e-Readers), de los cuales 9 son dispositivos (Kindle, Kobo, Bq, Sony Reader, Ectaco, jetBook, Aluratek, Woxter, iJoy) y 6 son aplicaciones (Kindle, Kobo, Google Play Books, iBooks, reportado en [3]. La ventaja de las aplicaciones consiste en la posibilidad de instalarlas en diversos dispositivos a diferencia de los dispositivos donde en la mayoría de ellos no se ofrece una versión funcional instalable fuera del dispositivo físico destinado para ello.

Se analizaron las características funcionales y se seleccionaron aquellas que pudieran ser de utilidad para el desarrollo de la PoC. Buscando su clasificación de la siguiente manera: la funcionalidad que permitiera la manipulación de textos como, cambios de fuente, subrayar, agregar notas se englobaron en una sección mientras que las funcionalidades que facilitaran la lectura y comprensión de textos como la posibilidad de consultar la definición de vocabulario, el registro del tiempo de lectura, la posibilidad de traducir parcial o totalmente el texto fueron englobadas en otra sección y por último se consideró si requerían la autenticación de usuario o si eran táctiles.

La PoC reportada en [5] inicialmente incluía la funcionalidad básica seleccionada del análisis realizado previamente. Dados los resultados obtenidos en [2], Donde se reportaron resultados de experimentos realizados en papel y donde se recomienda que las preguntas SC_P2 - SC_P7 y I_P0, I_P7 sean respondidas por medio de la recopilación de datos de IHC.

\section{Diseño}

Basados en esos resultados, se desarrolló una prueba de concepto inicial cuyo diseño es reportado en [5]. Esta prueba de concepto fue desarrollada como una aplicación Web, usando tecnologías HTML, CSS, y Angular JS para la interfaz.

El desarrollo siguió un Diseño Centrado en el Usuario (DCU) enfocándose hacia la información sobre las necesidades de usuarios. Se siguió un diseño cooperativo y participativo donde se trataron activamente a todas las partes involucradas [6]. En este caso los profesores interesados, participan en la definición de la interfaz de usuario en un proceso iterativo donde el llegar a acuerdos, priorizar necesidades y la comprensión de las mismas es la clave para un diseño exitoso.

La elaboración de la prueba de concepto se llevó a cabo bajo el desarrollo ágil software empleando específicamente la metodología scrum [7] lo cual permitió el avance y entrega significativos enfocándose a la satisfacción y necesidades prioritarias obteniendo retroalimentación en tiempo y forma sobre la funcionalidad propuesta.

\section{Resultados preliminares}

La prueba de concepto ha permitido verificar la captura de datos del proceso de lectura de comprensión de TTI. Algunos de estos datos son capturados por medio del registro de entradas de bitácora de las IHC que pueda ser presentada de manera cuantitativa para informar, en la medida de lo posible, del proceso de lectura y comprensión de una manera personalizada. Inicialmente las IHC se basaron principalmente en la funcionalidad de los e-Readers, y con el proceso iterativo de evaluación, esa funcionalidad ha sido adaptada a este proceso en específico. Por ejemplo, al momento de que un estudiante toca o da clic en una palabra, se despliega un menú que permite catalogar la información que el usuario necesita de esta palabra. Si el usuario accede a la palabra para leer su definición, se registra el número de ocurrencia de esta consulta así como el tiempo que tarda en consultarla.

También existe la posibilidad de que entienda la palabra pero no entienda el contexto en el que se usa, para solucionar este problema el sistema también capta el texto que el usuario resalta y lo registra en una base de datos facultando al docente para conocer la frase o párrafo que dificulta la comprensión del alumno y dirigirlo de mejor manera. Además si este texto es resaltado en común por un número significativo de estudiantes puede dar la pauta para que este sea verificado en clase por parte del profesor de carrera si así lo considera necesario. Al final, los datos que serán registrados en una base de datos podrán informar a los profesores y estudiantes interesados con la finalidad de tener un mejor entendimiento del proceso de lectura de cada estudiante y de definir mejores estrategias de enseñanza.

\section{Agradecimientos}

Un agradecimiento especial a la LEI Marcelina Pantoja Flores por su asesoría, amabilidad y aportaciones. A los profesores así cómo alumnos que participaron en las entrevistas. Juan Sebastián Pinto Orduz formó parte del proyecto de veranos de investigación científica en la Universidad de Guanajuato en la Sede de Yuriria. Mayra Yadira Mejia-Sierra agradece al Consejo Nacional de Ciencia y Tecnología (CONACyT) por el apoyo con la beca No. 649957.

\section{Referencias}

[1] Karahoca, A., Kanbul, S. and Marzban, A. 2011. World Conference on Information Technology Improvement of reading comprehension through computer-assisted language learning in Iranian intermediate EFL students. Procedia Computer Science. 3, (Jan. 2011), 3-10.

[2] Mejia Sierra, M.Y., Avila García, M.S., Bianchetti, M. and Vázquez de la Rosa, M.I. 2015. Análisis del proceso de la comprensión de textos técnicos en inglés. (Dec. 2015), 361372.

[3] Mejia Sierra, M.Y., Avila García, M.S., Bianchetti, M. and Vázquez de la Rosa, M.I. 2015. Diseño de un sistema de información para evaluación de la comprensión de textos técnicos en inglés. (Oct. 2015), 152-156.

[4] Moon, J.I. 2014. Groping for a new English teaching strategy. Pacific Science Review. 16, 4 (Dec. 2014), $207-$ 211.

[5] Pinto Orduz, J.S., Mejia Sierra, M.Y. and Avila García, M.S. 2016. Diseño y Desarrollo de una Prueba de Concepto de un Lector Electrónico (e-Reader) de Textos en Inglés. Jóvenes en la ciencia. 2, 1 (Jul. 2016), 11-15.

[6] Preece, J., Rogers, Y., Sharp, H., Benyon, D., Holland, S. and Carey, T. 1994. Human-Computer Interaction. AddisonWesley Longman Ltd.

[7] Pressman, R.S. and Troya, J.M. 1988. Ingeniería del software. McGraw Hill 